\title{
A Study on the Effects of Kalman Filter on Performance of IPMC-based Active Vibration Control Scheme
}

\author{
Dibakar Bandopadhya $^{1}$ and James Njuguna ${ }^{2 *}$ \\ ${ }^{1}$ Department of Mechanical Engineering, Indian Institute of Technology Guwahati \\ Guwahati, India \\ E-mail - dibakarb@iitg.ernet.in
}
${ }^{2}$ Centre for Automotive Technology, Department of Sustainable Systems, Cranfield University, Bedfordshire, MK43 0AL, UK
E-mail-j.njuguna@cranfield.ac.uk,

\begin{abstract}
This paper evaluates the effectiveness and performance of Ionic Polymer Metal Composite (IPMC) based active vibration control scheme equipped with the Kalman estimation algorithm. To assess the vibration attenuation efficiency, a rotating flexible manipulator has been modelled integrating two IPMC actuators following the modal approach. The elastic displacements as generalized coordinates for estimating optimal performance is carried out next by discretizing the elastic motion through the assumed mode technique and applying the Kalman filter. Simulations are then performed to demonstrate effective vibration attenuation using both IPMC and the Kalman filter. Kalman filter is employed for the whole vibrating system taking into account of the bending moment generated by the IPMC actuator. Experiment is conducted for the proposed damping scheme and the results are compared and verified with the simulation results.
\end{abstract}

Keywords: Active Vibration Control, Electro-active Polymer, Ionic Polymer Metal Composite, Heaviside Function

\section{Introduction}

A mechanical flexible link system is essentially a distributed parameter system with infinite degrees of freedom. The fundamental problem of actively controlling flexible link is to control a large dimensional system with a much smaller dimensional controller. For example, a large number of elastic modes may be needed to model the behaviour of a large flexible aero-blade; however, active control of all these modes is not possible due to computational and controlling limitations. Consequently, active control must be confined to few critical modes which are chosen according to the system performance requirements such as vibration tolerance and precision.

Apparently, the effect of the residual (uncontrolled) modes on the closed loop system is often ignored [1], although, effects of higher modes could be accommodated as disturbance in robust control.

Controls of rotational flexible links have been extensively studied in the past [2-5].

*Corresponding author: e-mail: j.njuguna@cranfield.ac.uk, phone: fax:
In space applications for instance, the robotic systems and vehicles are usually made of lightweight materials for effective performance and to help the launch. Unfortunately, these applications are subjected to a high degree of flexibility and generate unavoidable vibrations [6].

It is noteworthy that smart piezoelectric materials have been used successfully for controlling vibration of single and two link planar link systems [7-9]. On the other hand, links made of composite laminates exhibit nonproportional damping.

A hybrid control approach to control the gross motion of a rotational flexible link has been proposed by Sun et al. [10] using piezoelectric actuators (PZT) bonded on the surface of the flexible link to suppress the vibration. It was found that ferromagnetic materials show high damping at low strain levels (around 50-100 $\mu$-strain) only, however, for structural vibration control, induced strains are often at least one order higher in magnitude. To avoid this problem a hybrid damping scheme has been previously proposed in the literature [11-12]. Hybrid damping scheme is an interactive combination of active and passive damping [11]. Usually smart materials as 
finite dimension actuators are used to control the vibrations. However, with the introduction of magnetostrictive composites, such material can be used as distributed finite dimensional layers over the link to introduce distributed control of vibration. Smart magnetostrictive material layer like the Terfenol-D is used to achieve active damping in the link. The vibrational energy in these alloys is dissipated through magneto-elastic coupling. During vibration, the coatings of such materials undergo a change of strain, initiate a movement of magnetic domains and dissipate the mechanical energy through hysteresis [12].

Recently, the property of producing large bending moment or actuation strain by applying relatively low voltage has made IPMC attractive for both hybrid (strain dependent) and active damping applications. Ionic polymers require control voltage of around $3-5 \mathrm{~V}$ while piezoelectric actuators normally require around $200-500 \mathrm{~V}$. This helps to employ simple controller and electronics making ionic polymers a lightweight alternative. Further, due to high brittleness, PZT actuators are manufactured with relatively small dimension and may lead to breakdown under fatigue loading.

Electro-active polymers (EAP) belong to a class of active polymers that are divided into two categories, electronic, driven by electric field, and ionic, driven by diffusion of ions [13]. Ionic polymer material has both fixed anions and mobile cations. When the material is hydrated the cations will diffuse toward an electrode on the material surface under an applied electric field. Inside the polymer structure, anions are interconnected as clusters providing channels for the cations to flow towards the electrode. This motion of ions causes the structure to bend towards the anode [14-15]. The extremely large motion relative to size makes the polymers attractive as actuators. Also EAPs only require a few volts for actuation, usually less than $5 \mathrm{~V}[13,16]$. Ionic polymer metal composite (IPMC) are generally made of nafion, a perfluorinated polymer electrolyte, sandwiched in between platinum (or gold) electrodes on both sides [17-19].

The paper demonstrates a comparative study with an active vibration control scheme and a filtering technique. The main objective of the present studies is to correlate the performance of IPMC actuator to that of the Kalman filter algorithm for active vibration control. The comparison is important to demonstrate the vibration attenuation efficiency of IPMC with the real time estimator.

The proposed distributed PD controller using IPMC has been implemented with a flexible manipulator to assess the damping performance of the scheme. Due to low stiffness, the flexible manipulator generates parasite vibration during its movement. The vibration can be considered as the instantaneous dynamic tip deflection about its mean position. This vibrations cause errors in positioning the tip. The paper is organized by modeling the system and then employing the proposed PD control scheme with IPMC and then applying Kalman filtering algorithm. The damping performance of the system has been shown in terms of the reduction of the tip deflection (i.e. vibration) while in Kalman filter, the true states, that is, tip deflection and tip deflection rate of the system has been measured and subsequently measurement error identified. Because the feedback controller requires measurement of the states in order to achieve pointing accuracy, here, in the paper the states has been estimated continuously by using the Kalman filter and that subsequently relates the tip deflection of the controlled system.

A rotating flexible link has been modelled with two IPMC actuators to show the vibration suppression efficiency. The property of producing large bending deflection and subsequent bending moment of IPMC with low input voltage is utilized here. IPMC bonded to the flexible link bends and generates a bending moment that opposes the structural vibration. Two IPMC actuators are utilised for considering the first two modes of vibration. It is found that first two modes of the flexible link take the maximum amount of energy [20-21]. To meet the requirements for pointing accuracy; flexible body parameters such as elastic displacement are of great importance for control tasks and should be continuously identified in the working environment. Using the wellknown Kalman filter algorithm [22-24], the identification of flexible body parameters is found to be more efficient in the present study. Kalman filter gives the smallest possible estimation error by minimizing the 2-norm of the estimation error where the expected value of estimate is equal to the true state on an average. The theoretical results have been validated experimentally with a rotating flexible link using two IPMC actuators. Data acquisitions have been done through piezoelectric (bimorph) sensors located behind each IPMC and are used to record the strain amplitude in terms of voltage.

In section 2, IPMC properties are briefly discussed mainly focusing on the relationship between tip angle with input voltage. Section 3 presents the modelling of the flexible link with IPMC actuators. In section 4, Kalman filter estimation algorithm is discussed. Simulations and experiment results are discussed in section 5 and the conclusions are finally drawn.

\section{Bending Characteristics of IPMC}

In order to obtain the bending characteristics of IPMC with input voltage, an IPMC of size $(40 \times 5 \times 1) \mathrm{mm}^{3}$ has been tested in cantilever mode. Copper electrodes are used at one end and voltage is applied quasi-statically from a DC power supply (0-60V, 0-10A, supplied by Elnova Ltd., New Delhi) and subsequently deflection of IPMC is recorded through graph paper for each input voltage.

Euler-Bernoulli equation for the bent IPMC may be written as:

$M=\frac{E I}{R}=\frac{E I l}{R l}=\frac{E I}{l} \theta$

where, $M$ is the bending moment, $E I$ is the flexural rigidity of IPMC and $l$ is the length. $\mathrm{R}$ is the radius of curvature of IPMC for an input voltage V. From Figure 1, one can establish the voltage versus tip deflection relationship as:

$\theta=K_{1} V+K_{2}$ 
where, $\boldsymbol{K}_{\boldsymbol{1}}, \boldsymbol{K}_{2}$ are the constants. Using the experimental data, constants are evaluated using linear curve fitting approximation technique as shown in Figure 1 with a quality factor $\left(\mathrm{r}^{2}\right)$ of 0.9994 . Where, $K_{1}$ and $K_{2}$ are given by,

$$
\begin{aligned}
& K_{1}=\frac{\sum_{i=1}^{n} V_{i} \sum_{i=1}^{n} \theta_{i}-\sum_{i=1}^{n} V_{i} \theta_{i}}{\left\{\sum_{i=1}^{n} V_{i}\right\}^{2}-n \sum_{i=1}^{n} V_{i}^{2}} n=9 \\
& K_{2}=\frac{\left[\sum_{i=1}^{n} \theta_{i}-K_{1} \sum_{i=1}^{n} V_{i}\right]}{n}
\end{aligned}
$$

and the quality factor of the approximation is given by:

$$
r^{2}=K_{1}^{2} \frac{\left[\sum_{i=1}^{n} V_{i}^{2}-\frac{1}{n}\left(\sum_{i=1}^{n} V_{i}\right)^{2}\right]}{\left[\sum_{i=1}^{n} \theta_{i}^{2}-\frac{1}{n}\left(\sum_{i=1}^{n} \theta_{i}\right)^{2}\right]}
$$

$\theta=0.3347 V+0.0069 \quad 0.5 \leq V \leq 5$

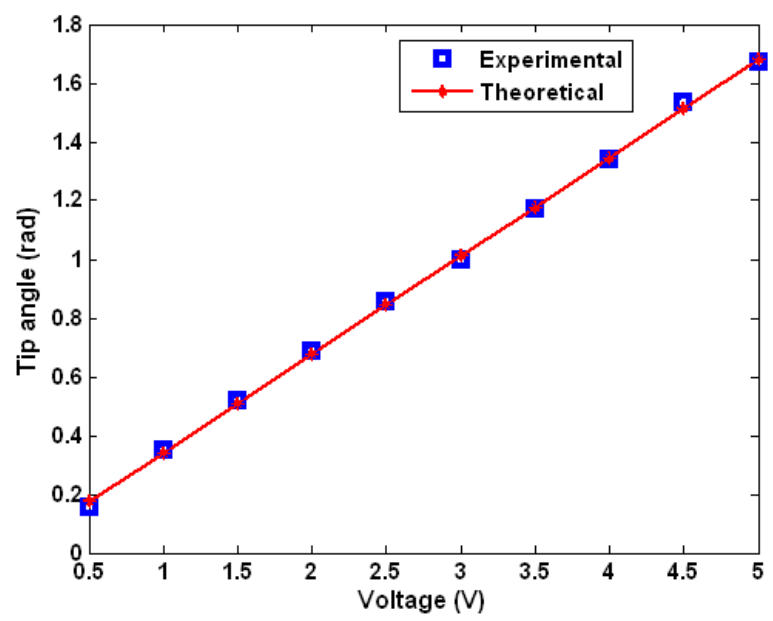

Figure 1. Change in tip angle of IPMC for various input voltage.

$K_{1}=0.3347$ and $K_{2}=0.0069$. Neglecting the constant $K_{2}$, equation (6) is simplified to:

$\theta=0.3347 \mathrm{~V}$

Therefore, bending moment generated by IPMC is obtained as:

$M=\left[0.3347 \frac{E I}{l}\right] V=K V$

where, the constant $K=0.3347 \frac{E I}{l}$. Figure 2 shows the variation of bending moment with input voltage.

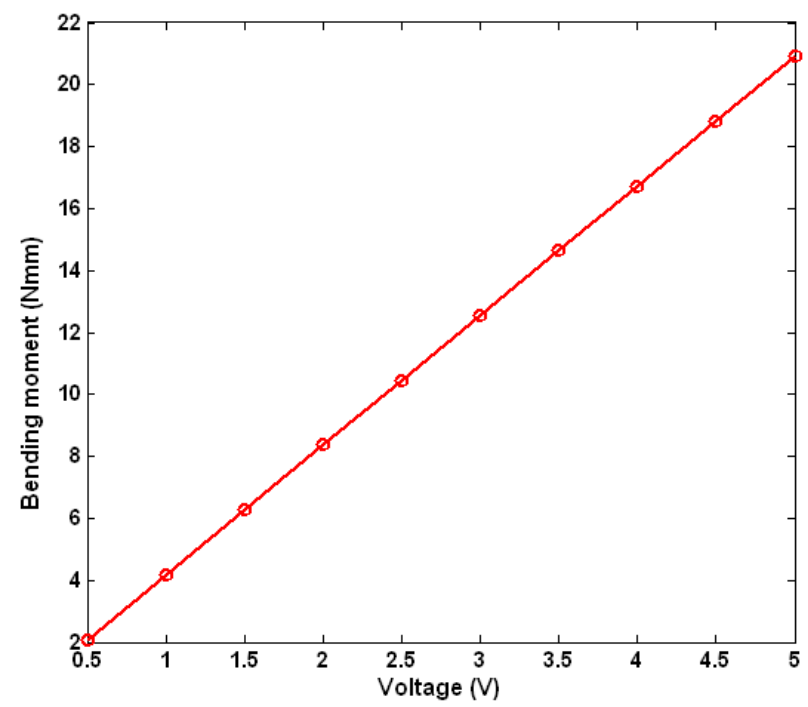

Figure 2. Experimentally obtained change in bending moment for various input voltages.

\section{Governing Equation of Motion with IPMC Patches}

The IPMC actuators bonded to the top surface of the flexible link are driven by low actuating voltage and generate distributed bending moment which opposes the structural vibration. Considering that uniform distributed bending moment generates over the region $x_{1}<x<x_{2}$, can be replaced in the link's differential equation by two point moments at $x_{1}$ and $x_{2}$ as shown in Figure 3, then, the governing equation of motion of a vibratory flexible link can be expressed as:

$\frac{\partial^{2}}{\partial x^{2}}\{M(x)-m(x)\}+\rho_{b} \frac{\partial^{2}}{\partial t^{2}} w(x, t)=T$

where, $w(x, t)$ is the transverse displacement of the flexible link, $M(x)$ is the beam internal bending moment, $m(x)$ is the externally applied distributed bending moment from IPMC actuator, $T$ is the motor torque supplied at the hub and $\rho_{\boldsymbol{b}}$ is the mass per unit length of the flexible link.

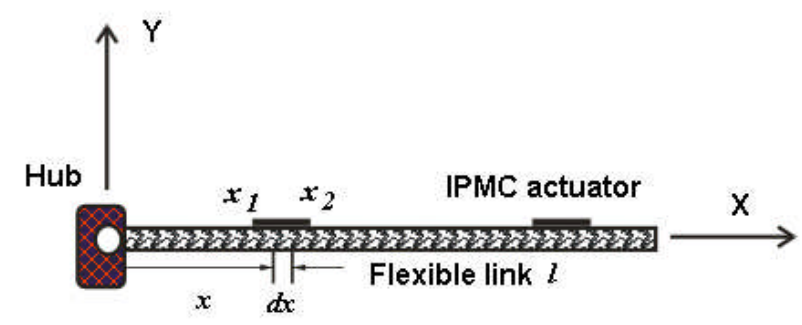

Figure 3. Placement of IPMC patches along the length of the flexible link (top view).

The external bending moment can be expressed as: 
$m(x)=\left\{\begin{array}{l}K V \quad x_{1}<x<x_{2} \\ 0 \quad \text { otherwise }\end{array}\right.$

This expression can be expressed in terms of Heaviside Function as:

$m(x)=K V\left[H\left(x-x_{1}\right)-H\left(x-x_{2}\right)\right]$

where, $H(x)=\left\{\begin{array}{lll}0 & \text { if } & x<0 \\ 1 & \text { if } & x>0\end{array}\right.$, therefore, introducing step function one can write the equation as:

$\frac{d^{2}}{d x^{2}} m(x)=K V \frac{d^{2}}{d x^{2}}\left[H\left(x-x_{1}\right)-H\left(x-x_{2}\right)\right]$

$=K V\left[\delta^{\prime}\left(x-x_{1}\right)-\delta^{\prime}\left(x-x_{2}\right)\right]$

Therefore, substituting all values of $M(x)$ and $m(x)$ in equation (9) one can obtain the equation of motion as,

$E_{b} I_{b} \frac{\partial^{4} w}{\partial x^{4}}+\rho_{b} \frac{\partial^{2} w}{\partial t^{2}}=$

$T+K V\left[\delta^{\prime}\left(x-x_{1}\right)-\delta^{\prime}\left(x-x_{2}\right)\right]$

Equation (13) is describing the motion of the distributed parameter system. In order to obtain a closed form solution, a modal decomposition of the form can be considered as:

$w(x, t)=\sum_{i=1}^{\alpha} \phi_{i}(x) q_{i}(t)$

where, $\phi_{i}(x)$ are the mode shapes and $q_{i}(t)$ are the time dependent modal amplitudes, considering first finite ' $\mathrm{n}$ ' modes then equation (13) is transformed into,

$E_{b} I_{b} q_{i}(t) \phi_{i}^{\prime \prime \prime \prime}(x)+\rho_{b} \phi_{i}(x) \ddot{q}_{i}(t)=$

$T+m\left[\delta^{\prime}\left(x-x_{1}\right)-\delta^{\prime}\left(x-x_{2}\right)\right]$

Multiplying by both sides by $\phi_{i}(x)$ and integrating along the length $l$ of the link equation (15) gives

$\int_{0}^{l} \rho_{b} \phi_{i}(x)^{2} \ddot{q}_{i}(t) d x+\int_{0}^{l} E_{b} I_{b} \phi_{i}(x) \phi_{i}^{\prime \prime \prime}(x) q_{i}(t) d x$

$=\int_{0}^{l} T \phi_{i}(x) d x+m \int_{0}^{l} \phi_{i}(x)\left[\delta^{\prime}\left(x-x_{1}\right)-\delta^{\prime}\left(x-x_{2}\right)\right] d x$

In figure 4 , it is shown that the first two modes take the maximum amount of energy and on increment of mode numbers total energy does not change appreciably. Based on this we have considered two strips of IPMC as actuators as it will be satisfactory for suppression of the two major modes of vibration. This will also minimize the cost of IPMC being used.

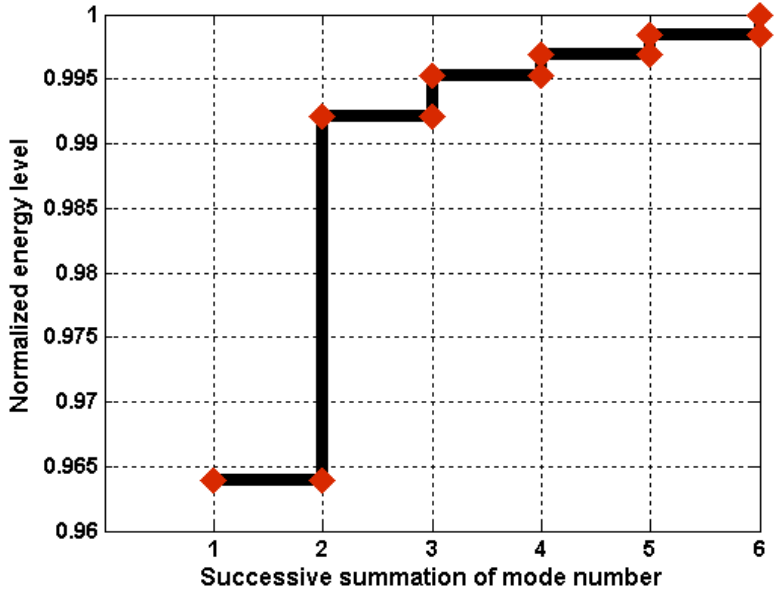

Figure 4. Successive summation of total energy as mode number increases up to six.

For normalized mode shape, taking $\int_{0}^{l} \phi_{i}(x)^{2} d x=1$, equation (16) is further reduced to,

$$
\begin{aligned}
& \rho_{b} \ddot{q}_{i}(t)+E_{b} I_{b} q_{i}(t) \psi_{i}= \\
& T \int_{0}^{l} \phi_{i}(x) d x+K V\left[\phi_{i}^{\prime}\left(x_{2}\right)-\phi_{i}^{\prime}\left(x_{1}\right)\right]
\end{aligned}
$$

where, $\psi_{i}$ determines the modal stiffness and is given by $\psi_{i}=\int_{0}^{l} \phi_{i}(x) \phi_{i}^{\prime \prime \prime \prime}(x) d x$ and introducing the hub inertia one can rewrite the equation as:

$$
\begin{aligned}
& I_{h} \ddot{\theta}+\rho_{b} \ddot{q}_{i}(t)+E_{b} I_{b} q_{i}(t) \psi_{i}= \\
& T \int_{0}^{l} \phi_{i}(x) d x+K V\left[\phi_{i}^{\prime}\left(x_{2}\right)-\phi_{i}^{\prime}\left(x_{1}\right)\right] \\
& I_{h} \ddot{\theta}+\rho_{b} \ddot{q}_{i}(t)+E_{b} I_{b} \psi_{i} q_{i}(t)=Q_{i} T+R_{i} V
\end{aligned}
$$

Define, $K_{q_{i}}=\Omega_{i}^{2}=E_{b} I_{b} \psi_{i}$,

$R_{i}=\left[\phi_{i}^{\prime}\left(x_{2}\right)-\phi_{i}^{\prime}\left(x_{1}\right)\right] K, Q_{i}=\int_{0}^{l} \phi_{i}(x) d x$ the system governing equation is simplified to:

$$
I_{h} \ddot{\theta}+\rho_{b} \ddot{q}(t)+K_{q} q(t)=Q T+R V
$$

Equation (20) can be written in simplified form as,

$\left[\begin{array}{cc}0 & 0 \\ I_{h} & \rho_{b}\end{array}\right]\left[\begin{array}{l}\ddot{\theta} \\ \ddot{q}\end{array}\right]+\left[\begin{array}{cc}0 & 0 \\ 0 & K_{q}\end{array}\right]\left[\begin{array}{l}\theta \\ q\end{array}\right]=\left[\begin{array}{ll}0 & 0 \\ Q & R\end{array}\right]\left[\begin{array}{l}T \\ V\end{array}\right]$

$M \ddot{\Theta}+K_{s} \Theta=B_{f} U$ 
where, the state vector $\Theta=\left[\begin{array}{l}\theta \\ q\end{array}\right]$, input matrix $B_{f}=\left[\begin{array}{ll}0 & 0 \\ Q & R\end{array}\right]$ and $K_{s}=\left[\begin{array}{cc}0 & 0 \\ 0 & K_{q}\end{array}\right]$ is the stiffness matrix. It is to be noted that $\mathrm{i}$-th mode is controllable if and only if $B_{f}$ is nonzero.

\section{Kalman Filter}

The proposed feedback control system always requires the measurement of tip deflection and tip deflection rate of actual system. Further potential noise immunity also requires careful filter design. In the proposed Kalman filter, the noise has been introduced in the actual system, as the derivative gain is highly susceptible to noise. In addition, sometimes due to input to the system, nonlinearity and high frequency modal terms, the system response becomes oscillatory. These can be minimized by filtering the output response of the feedback controller i.e. the measurement data.

The Kalman filter is a computational algorithm containing a sequence of time and measurement updating of the estimates of the system. It is used to identify the elastic displacements or system identification parameters. The Kalman filter can incorporate dynamic noise in the dynamical model of the state. It is a real time estimator supplying the estimates for the instant that the measurement is available. The filter algorithm is essentially a set of mathematical equations that implement a predictioncorrector type estimator that is optimal in the sense that it minimizes the estimated error covariance when some presence conditions are met. The many approaches to this basic problem are typically based on the state-space model. There is typically a process model that models the transformation of the process state. This can usually be represented as a linear stochastic difference equation as in equation (23). The filter consists of two cycles [25-26]:

- Time Update

- Measurement Update

\subsection{State dynamics model}

The state dynamical model can be represented as

$x=A x+B u+w$

where, $x=\left(\begin{array}{llll}\theta & q & \dot{\theta} & \dot{q}\end{array}\right)^{T}$ is the state continuously variant

in the time, $B$ is the input matrix in addition of process noise $w$ and $A$ is the system matrix that relates the state timely and linearly and is given by

$A=\left[\begin{array}{r|l}0 & I \\ \hline-K_{A} & -C_{A}\end{array}\right]$

where, $K_{A}=\operatorname{diag}\left\{0, \omega_{n}{ }^{2}\right\}$, the matrix contains the squared natural frequencies, and the damping matrix $C_{A}=\operatorname{diag}\left\{0,2 \xi \omega_{n}\right\}$. The natural frequencies of the first three modes are given by: $\omega_{n_{1}}=32.34 \mathrm{rad} / \mathrm{sec}, \quad \omega_{n_{2}}=202.5504 \mathrm{rad} / \mathrm{sec}$ and $\omega_{n_{3}}=567.1505 \mathrm{rad} / \mathrm{sec}$. Further the damping coefficient for the aluminium is taken as: $\xi=0.01$.

\subsection{Measurement model}

In addition of the above model, there is some form of measurement model that describes the relationship between the process state and the measurements. This can usually be represented with a linear expression. The measurement model is given by:

$y=H x+v$

where, $y$ is the measurement vector composed by the

angle $\theta$ and angular velocity $\dot{\theta}$ observables. The measurements of $\theta$ and $\dot{\theta}$ are taken by a position and velocity sensor. For the angular position and velocity it is assumed nominally a standard deviation of $0.1^{\circ}$ and $0.01^{\circ} / s$ respectively. The $H$ matrix relates the measurements to the state by

$H=\left[\begin{array}{llll}1 & 0 & 0 & 0 \\ 0 & 0 & 1 & 0\end{array}\right]$

where, $v$ represents the measurement noise. The specific equations for the time and measurement updates are presented below. Figure 5 shows the schematic diagram of the Kalman filter for estimating the state variables of the flexible link.

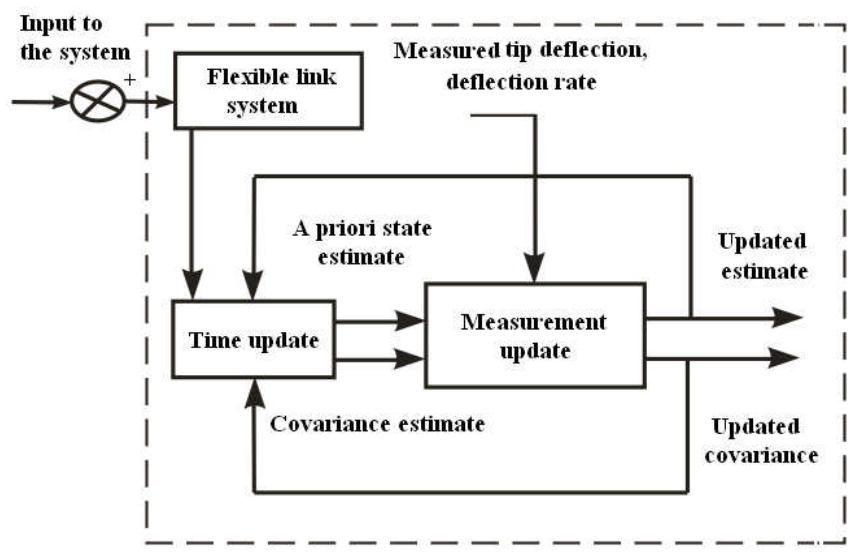

Figure 5. A schematic diagram of the Kalman filter designed to estimate the state vector of the flexible link.

The time update, is represented as

$$
\begin{aligned}
& \bar{x}_{k}=A x_{k-1}+B u_{k-1} \\
& \bar{P}_{k}=A P_{k-1} A^{T}+Q
\end{aligned}
$$

while the measurement update

$$
K_{k}=\bar{P}_{k} H^{T}\left(H \bar{P}_{k} H^{T}+R\right)^{-1}
$$


$\stackrel{+}{x}_{k}=\bar{x}_{k}+K_{k}\left(y_{k}-H \bar{x}_{k}\right)$

$P_{k}=\left(1-K_{k} H\right) \bar{P}_{k}$

where,

$\bar{x}_{k} \in R^{n}$ : Priori state estimate at step $\mathrm{k}$,

$x_{k} \in R^{n}$ : Posteriori state estimate at step $\mathrm{k}$,

$\bar{P}_{k} \in R^{n}$ : Priori estimate error covariance at step k,

$P_{k} \in R^{n}$ : Posteriori estimate error covariance at step k.

$Q$ : Process noise covariance,

$R$ : Measurement noise covariance,

$y_{k}-H \bar{x}_{k}$ : The measurement innovation or residual.

The first task during the measurement update is to compute the Kalman Gain $K_{k}$. After each time and measurement update pair, the process is repeated with the previous posteriori estimates used to project or predict the new a priori estimates.

\section{Results and Discussion \\ 5.1. Simulations}

In this section, the results obtained from numerical simulation of single-link flexible manipulator using the material properties as shown in Table 1. All programs are developed in MATLAB. To solve the differential equations in state space, the in-built function of MATLAB called "ode45" is used. Ode45 is based on 4-th and 5-th order Runga-Kutta scheme with adaptive step size. Square torque of magnitude $1 \mathrm{Nm}$ is used for simulation that is applied to the link via a stepper motor.

\begin{tabular}{|c|c|c|}
\hline Property & Aluminium & IPMC \\
\hline Elastic modulus & $70 \mathrm{GPa}$ & $1.2 \mathrm{GPa}$ \\
\hline Length & $0.8 \mathrm{~m}$ & $40 \mathrm{~mm}$ \\
\hline Width & $50 \mathrm{~mm}$ & $5 \mathrm{~mm}$ \\
\hline Thickness & $4 \mathrm{~mm}$ & $1 \mathrm{~mm}$ \\
\hline Area of c/s & $200 \mathrm{~mm}^{2}$ & $5 \mathrm{~mm}^{2}$ \\
\hline Density & $2700 \mathrm{Kg} / \mathrm{m}^{3}$ & $2000 \mathrm{Kg} / \mathrm{m}^{3}$ \\
\hline
\end{tabular}

Table 1. Material and physical properties of both link and IPMC.

Two patches of IPMC are used to actively damp the vibration of flexible link. A distributed active strain is generated by driving the required voltage through the IPMC when it is used as active damper [21-22]. Figure 6 shows both the undamped and damped response of the flexible link for square torque input. It is observed that using two patches of IPMC around $80 \%$ of tip deflection reduction is possible as shown in Figure 7. Thus, it is observed that the proposed vibration control scheme attenuate vibration considerably.

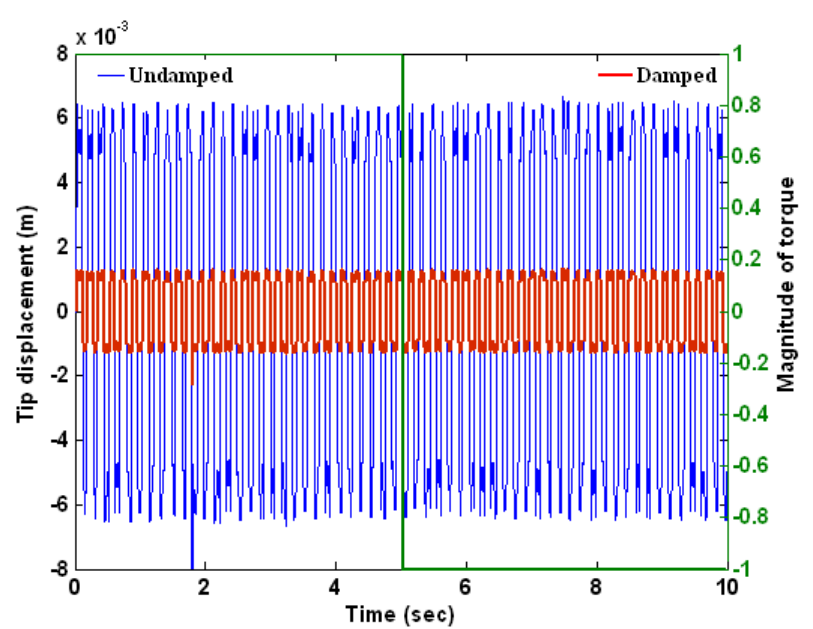

Figure 6. Undamped and damped vibration response of the flexible link for square torque input of magnitude $1 \mathrm{Nm}$.

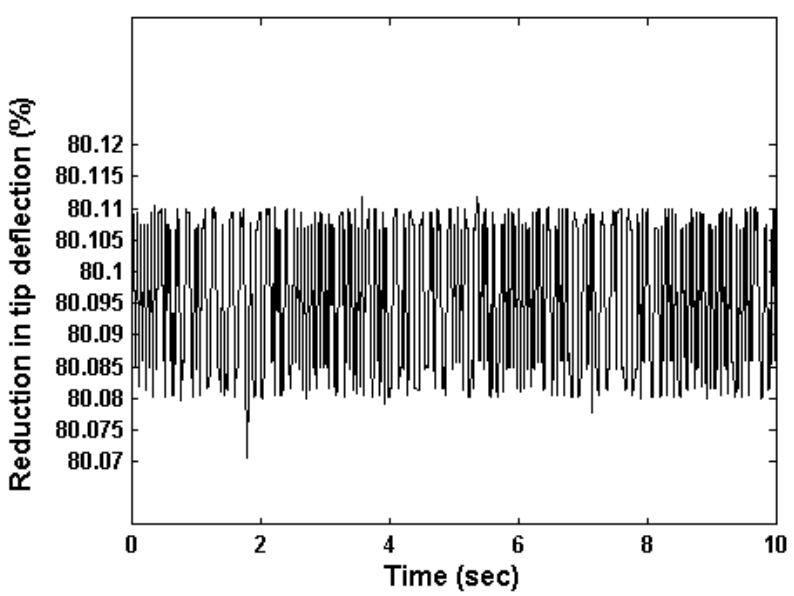

Figure 7. Reduction in tip deflection of the flexible link during active damping.

In the follow-up, Kalman filter estimates the tip deflection and tip deflection rate of the flexible link for square torque input of magnitude $1 \mathrm{Nm}$. Filter's performance is assessed for a time step of $0.0067 \mathrm{sec}$ while total time is taken 10 seconds to estimate the system variables. As the proposed control scheme susceptible to noise, therefore, noises are introduced in the actual system in order to estimate the true states of the flexible manipulator. Figure 8 shows the actual tip deflection with the filtered tip deflection of the flexible manipulator about its mean axis. The true response of the system represents the combined vibration and noises of the system. Figure 9 separately illustrates error filtered in terms of tip deflection of the flexible link. Similarly, Figure 10 shows the actual response and filtered response of the deflection rate of the link while Figure 11 shows the error filtered. It is found that relative error of tip deflection and tip deflection rate is $7.2 \%$ and $7.44 \%$ respectively. 


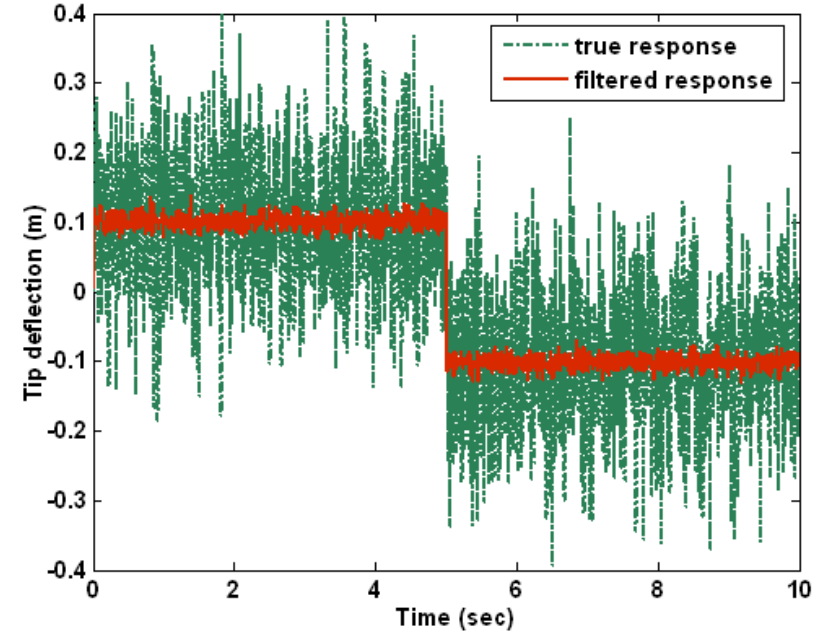

Figure 8. True response and estimated response of the flexible link for square torque input.

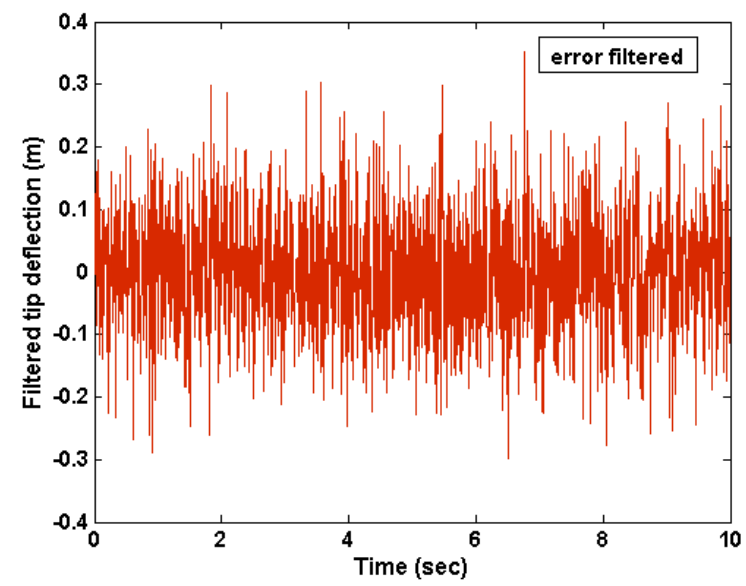

Figure 9. Error for filtered tip deflection using for square torque input.

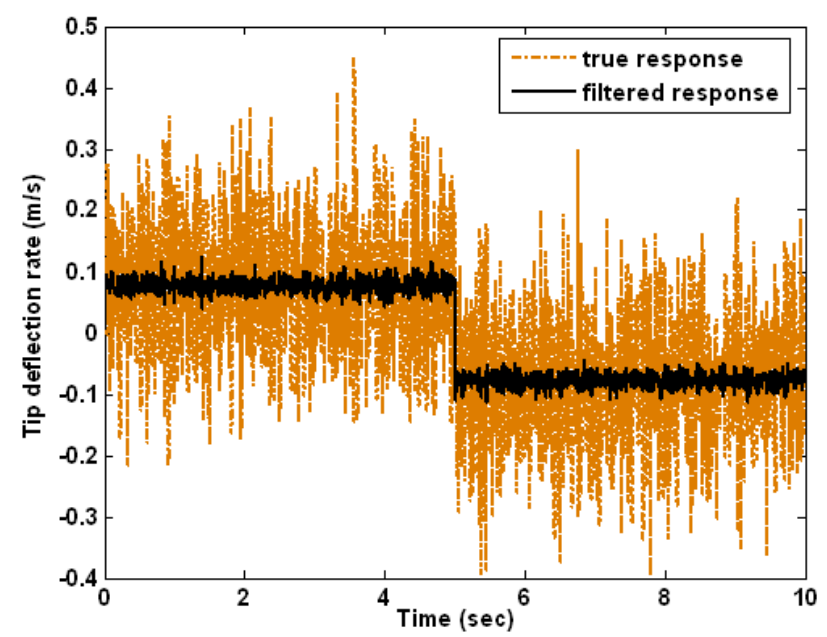

Figure 10. True tip deflection rate and estimated deflection rate for square torque input.

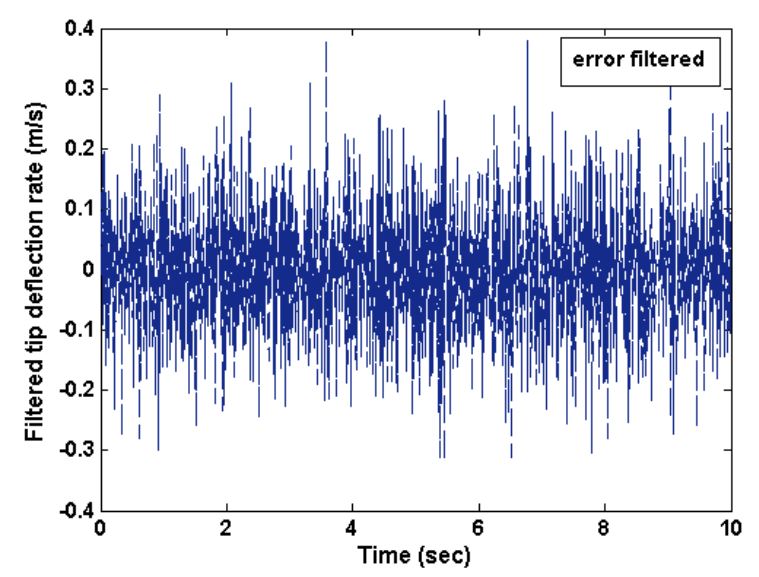

Figure 11. Error filtered for rate of tip deflection.

\subsection{Experiments}

In order to validate the damping performance of IPMC actuator with the Kalman filter, extensive experiment has been conducted using IPMC with a flexible manipulator with different amplifier gain.

Kalman filter is used for attaining the vibration response of the whole system i.e. flexible manipulator with IPMC. Where bending moment obtained through experiment is incorporated in the estimation algorithm and subsequently the tip deflection is measured. While experiment is conducted using the proposed distributed active vibration control scheme. Subsequently the effectiveness of the active vibration control scheme is compared with the filtered results.

The experiment is done with a rotating flexible link of length $80 \mathrm{~cm}$ made of aluminium and is rotated by a stepper motor. Two IPMC actuators are placed at $30 \mathrm{~cm}$ and $60 \mathrm{~cm}$ away from the hub end with two piezoelectric (bimorph) sensors placed collocatedly behind each of the IPMC $\left(40 \times 10 \times 0.2\left(\mathrm{~mm}^{3}\right)\right)$ actuators to develop two independent proportional derivative (PD) control loops as shown on Figure 12. Figure 13 shows the experiment setup with two IPMC actuators with other control accessories.

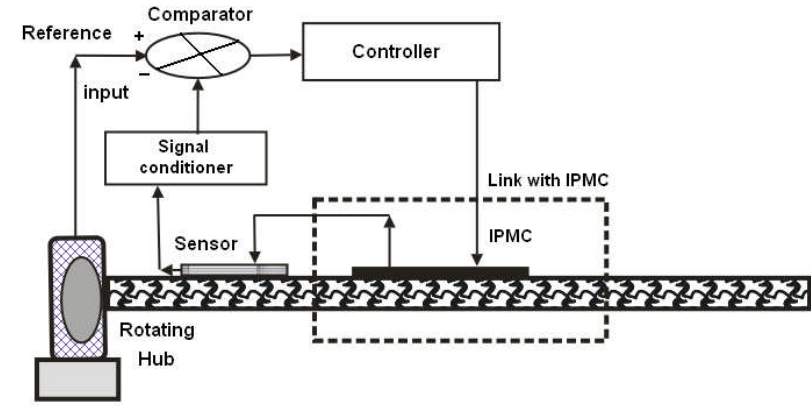

Figure 12. Schematic diagram (top view) for the flexible link system with an independent single loop PD controller for active vibration control.

The details of the controller design are discussed in Ref. [21]. Sensor data is collected by PC interfaced DAQ-PCI6251 and NI-DAQmx (supplied by National 
Instrumentation) with signal conditioner. The flexible link is rotated with constant torque in to-and-fro fashion with a stepper motor within 72 degrees angular position to vibrate the link. The operating speed of the motor is kept $30 \mathrm{rpm}$ with 3.6 degrees step size. The cyclic motion of the flexible link along with the stepper motor's step motion generates vibration. LabView software is employed to study and analyze the vibration. Through Fast Fourier Transform of the sensors data of undamped system, it is observed that the link vibrates predominantly with only lower modes of vibration around $5-40 \mathrm{~Hz}$. Higher modes of excitation up to $120 \mathrm{~Hz}$ is also observed. The working principle is based on the bimorph signal sensed and the IPMC is actuated accordingly so as to generate a bending moment to oppose vibration. Experimentally, it has been verified that the voltage generated in the bimorph sensor is proportional to the tip deflection. Figure 14 shows the PD controller used in the present experiment for vibration control. The response of the system using PD controller with positive unity feedback can be expressed as:

$\frac{C(s)}{R(s)}=\frac{\left(K_{P}+K_{D} s\right) G(s)}{1+\left(K_{P}+K_{D} s\right) G(s)}$. Where, $G(s)$ is the transfer function of the flexible manipulator with IPMC and bimorph. Theoretically, the transfer function of the flexible manipulator is obtained as: $G(s)=\frac{1}{s^{2}+2 \xi \omega s+\omega^{2}}$

where, $\xi$ and $\omega$ are the damping factor and the modal frequency respectively. The experiment is conducted using the following PD compensator gain:

Proportional gain $\left(K_{P}\right)=1.5$, Derivative gain $\left(K_{D}\right)=0.14$.

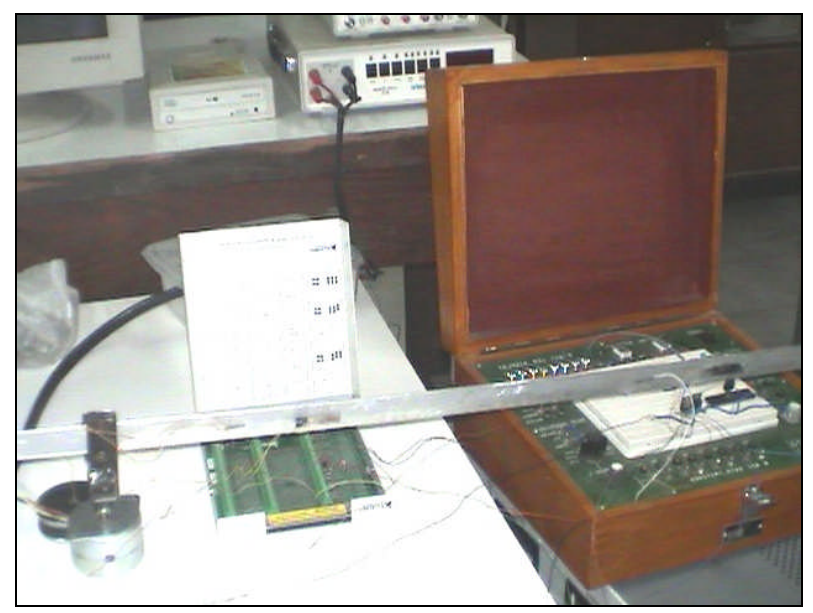

Figure 13. Experiment setup is showing flexible link with two IPMC actuators.

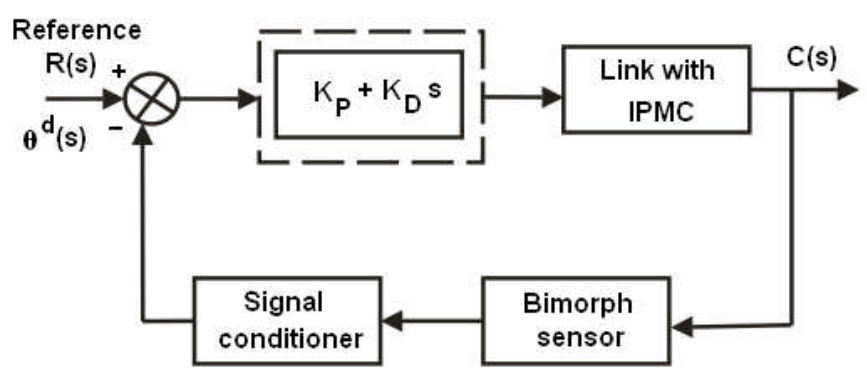

Figure 14. PD controller used in the proposed vibration control [21].
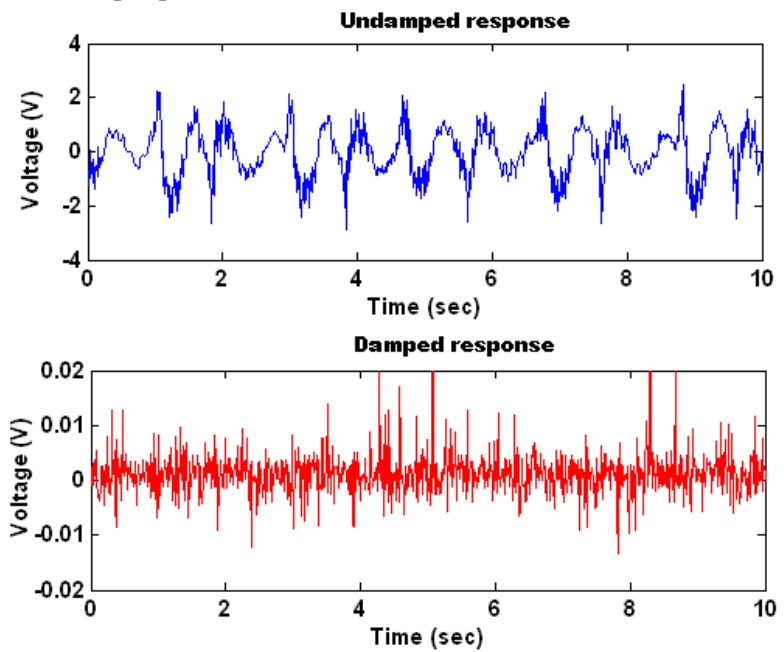

Figure 15. Undamped (top) and damped (bottom) vibration response of the flexible link recorded in sensor 1 during rotation using two patches of IPMC with amplifier gain $=1$.

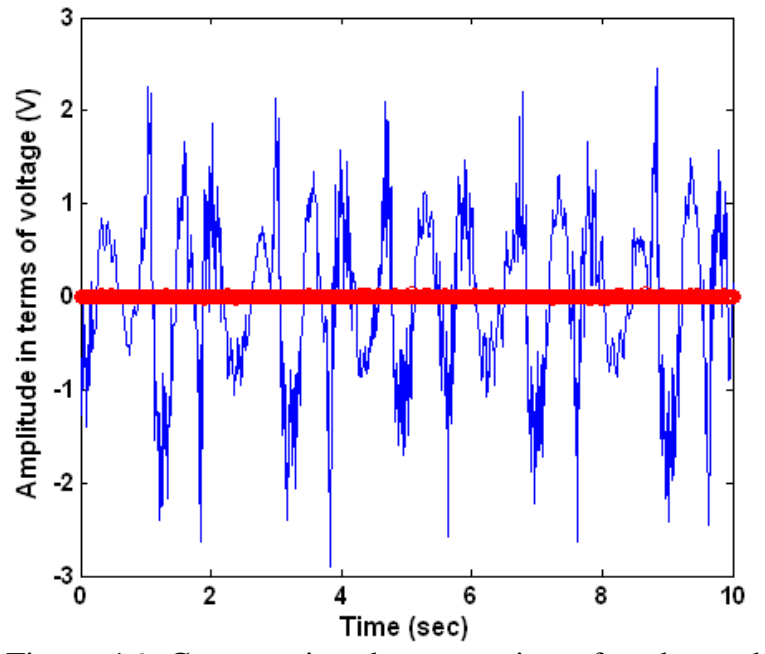

Figure 16. Comparative demonstration of undamped and damped vibration response recorded in sensor 1 . 


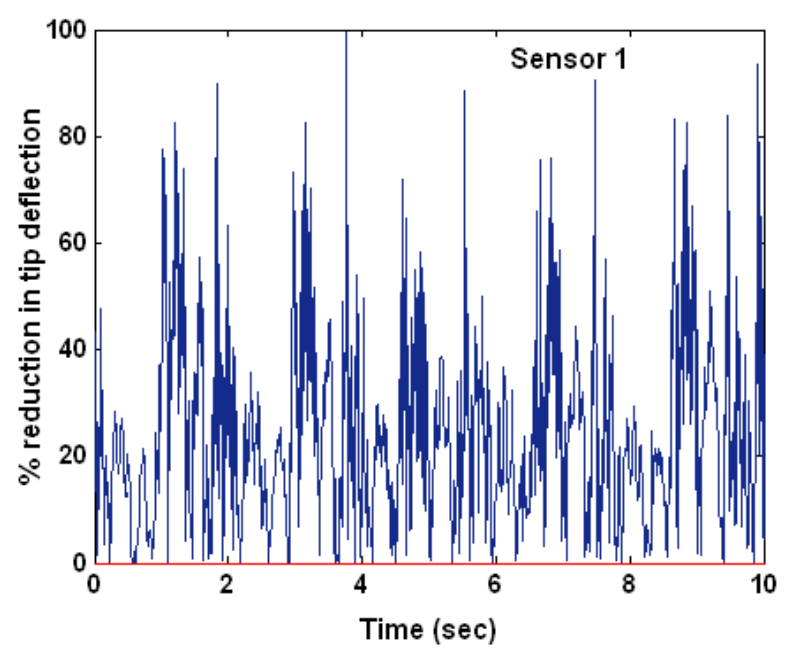

Figure 17. Percentage reduction in tip deflection in sensor 1.
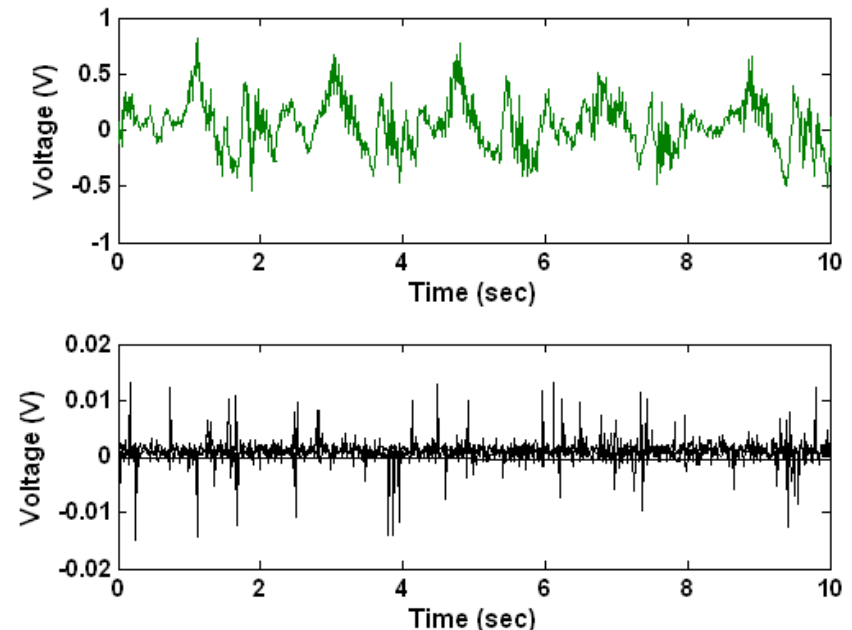

Figure 18. Undamped (top) and damped (bottom) vibration response of the flexible link recorded in sensor 2 during rotation using two patches of IPMC with amplifier gain $=1$.

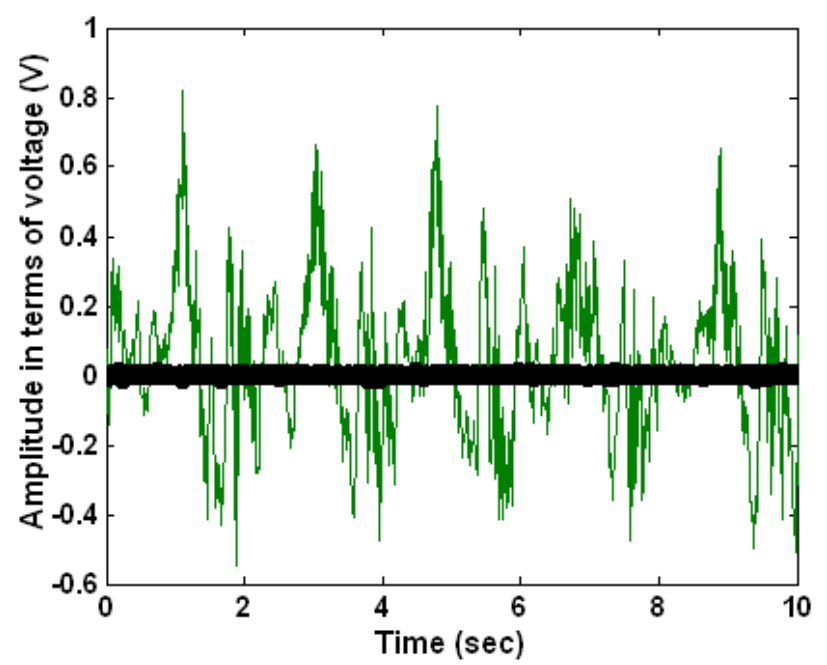

Figure 19. Comparative evaluation of damped and undamped vibration response recorded in sensor 2 .
Figure 15 illustrates the undamped and damped vibration response of the flexible link recorded in sensor 1 while Figure 16 shows the comparative evaluation of the proposed active damping scheme. Figure 17 illustrates the corresponding percentage reduction in tip deflection in sensor 1. It is observed from the experiment results that vibrations have been attenuated considerably. Figure 18 shows the undamped and damped vibration response of the flexible link recorded in sensor 2 while Figure 19 shows the comparative evaluation of damping experiment in sensor 2. Figure 20 and Figure 21 demonstrate the Fast Fourier Transform of the undamped response data recorded in sensor 1 and sensor 2 respectively. It is observed that the bandwidth of the vibration remain within $10-80 \mathrm{~Hz}$. Through experiment, it is observed that up to $60-80 \%$ reduction in tip deflection of flexible link is achievable.

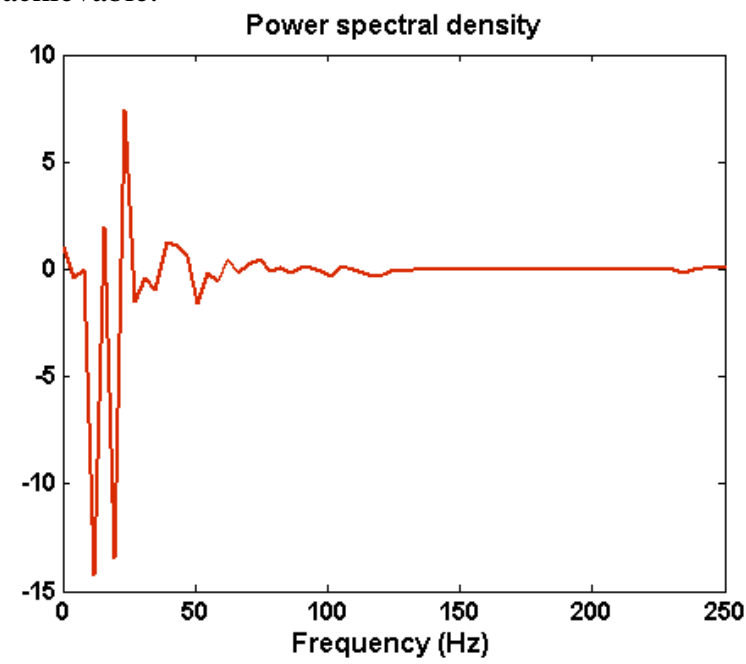

Figure 20. Fast Fourier Transform (FFT) of the undamped vibration response of the flexible link recorded in sensor 1.

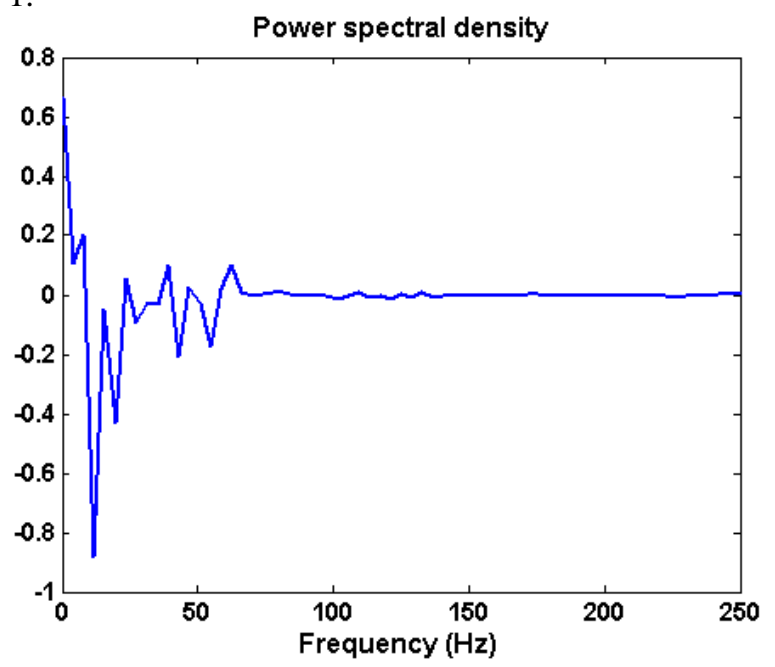

Figure 21. Fast Fourier Transform (FFT) of the undamped vibration response of the flexible link recorded in sensor 2.

\section{Conclusion}


The contribution of the paper is to verify the contribution to the effectiveness of IPMC actuator with the Kalman filter for controlling vibration. Kalman filter algorithm is found fruitful to estimate the tip deflection and tip deflection rate of the link and based on which comparison is made with the proposed vibration control scheme. It is observed that incrementing IPMC actuators are a good means to attenuate the high amplitude vibration more effectively. This would of course increase the cost of control.

\section{References}

[1] M.J. Balas, "Active Control of Flexible System", Journal of Optimization Theory and Applications, Vol. 25, No. 3, 1978.

[2] Moudgal V G, Passino K M and Yurkovich S 1994 "Rule-based control for a flexible-link robot", IEEE Trans. Control Syst. Technol. vol. 2, no. 4, pp.392-405.

[3] Choi S B, Cheong C C and Shin H C 1995 "Sliding mode control of vibration in a single-link flexible arm with parameter variations", J. Sound Vib. Vol.179, no. 5, pp. 737748.

[4] Morita Y, Ukai H and Kando H 1997, "Robust trajectory tracking control of elastic robot manipulators", ASME J. Dyn.Syst. Meas. Control vol. 119, no. 4, pp. 727-735.

[5] Yang S M, Jeng J A and Liu Y C 1997, "Controller design of a distributed slewing flexible structure: a frequency domain approach", ASME J. Dyn. Syst. Meas. Control vol. 119, no. 4, pp. 809-814.

[6] Yang J H, Lian F L and Fu L C 1997, "Nonlinear adaptive control for flexible-link manipulators", IEEE Trans. Robot.Autom. vol. 13, no. 1, 140-148.

[7] Kim Hyoung-Kyu, Choi Seung-Bok and Thompson Brian S. 2001. "Compliant control of a two-link flexible manipulator featuring piezoelectric actuators", Mechanism and Machines Theory, vol. 36, no. 3, pp.411-424.

[8].Ho Cheol Shin and Seung Bok Choi 2001. "Position control of a two link flexible manipulator featuring piezoelectric actuators and sensors", Mechatronics, Vol. 11(6), pp. 707-729.

[9] Dong Sun, James K. Mills, Jinjun Shan, S.K. Tso, “A PZT actuator control of a single-link flexible manipualtor based on linear velocitry feedback and actuator palcement", Mechatronics 14 (2004) 381-401.

[10] Dong Sun, Jinjun Shan, Yuxin Su, Hugh H T Liu, Chiming Lam, "Hybrid control of rotational flexible link using enhanced PD feedback with a nonlinear differentiator and PZT actuators", Smart Mater. Struct. vol. 14, no. 1, pp. 69-78, 2005.

[11] Bhattacharya B., Vidyashankar B.R., Patsias S. and Tomlinson G.R. 2000, "Active and passive vibration control of flexible structures using a combination of magnetostrictive and ferro-magnetic alloys", Proceedings of SPIE fifth European Conference on Smart Structures and Materials, vol. 4073, pp.204-214.

[12] Dibakar Bandopadhya, Bishakh Bhattacharya, Ashish Dutta, "Modeling of Hybrid Damping Scheme Using Smart Magnetostrictive Composites for Flexible Manipulator",
Journal of Reinforced Plastics and Composites, 2007, 26(9) 861-880.

[13] M. Shahinpoor, K. J. Kim, "Ionic Polymer Metal Composites Fundamentals -I", Smart. Mater. Struct. vol. 10, no. 4, pp. 819-833, 2001.

[14] Kim K J and Shahinpoor M 2003 "Ionic Polymer Metal Composites -II Manufacturing Technique", Smart Materials and Structure", vol. 12, no. 1, pp. 65-79.

[15] M. Shahinpoor, K. J. Kim, "Ionic polymer-metal composites: III. Modeling and simulation as biomimetic sensors, actuators, transducers, and artificial muscles", Smart Mater. Struct., vol. 13, no. 6, pp.1362-1388, 2004.

[16] M. Shahinpoor, K. J. Kim, "Ionic polymer-metal composites: IV. Industrial an dmedical applications", Smart Mater. Struct., vol. 14, no. 1, pp. 197-214, 2005.

[17] J.W. Paquette, K.W. Kim, D. Kim, W. Yim, "The behavior of ionic polymer-metal composites in a multilayer configuration", Smart Mater. Struct. vol. 14, no. 5, pp. 881-888, 2005.

[18] Deuk Yong Lee, Seok Park, Myung-Hyun Lee, Kwang J. Kim, Seok Heo, "Ionic polymer-metal composite bending actuator loaded with multi-walled carbon nanotubes", Sensors and Actuators, vol. 133, no. 1, pp. 117-127, 2007.

[19] M. Shahinpoor, K.J. Kim, "The effect of surface electrode resistance on the performance of ionic-polymer metal composite (IPMC) artificial muscles", Smart Mater, Struct. vol. 9, no. 4, pp. 543-551, 2000.

[20] Dibakar Bandopadhya, et al. "An Active Vibration Control Strategy for a Flexible Link Using Distributed Ionic Polymer Metal Composites", Smart Materials and Structures, 2007, 16(3) 617-625.

[21] Dibakar Bandopadhya, Bishakh Bhattacharya, Ashish Dutta, "Active vibration control strategy for a single link flexible manipulator using ionic polymer metal composites", Journal of Intelligent Material Systems and Structures, vol. 19, No. 4, pp. 487-496, 2008.

[22] Trigolo Kuga, De Souza, "Parameter Identification of a Rigid Flexible Satellite Using Kalman Filter", Proceedings, COBEM, November 10-14, Sao Paulo, 2003.

[23] D. Simon, D.L. Simon, "Aircraft Turbofan Engine Health Estimation Using Constrained Kalman Filtering", ASME Journal of Engineering for Gas Turbines and Power, Vol. 127, no. 2, pp. 323-328, April 2005.

[24] D. Simon, H. El-Sherief, "Hybrid Kalman/Minimax Filtering in Phase Locked Loops", Control Engineering Practice, Vol. 4, pp. 615-623, October 1996.

[25] D. Simon, "Kalman Filtering for fuzzy discrete time dynamic systems", Applied Soft Computing, Vol. 3, pp. 191-207, 2003.

[26] B. Anderson and J. Moore, "Optimal Filtering", Prentice-Hall, 1979. 


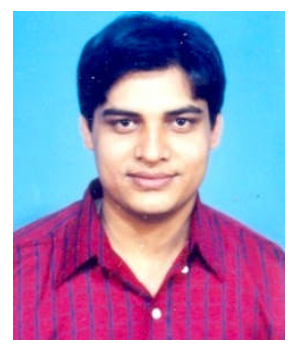

Dibakar Bandopadhya is currently working as Assistant Professor, Department of Mechanical Engineering, Indian Institute of Technology Guwahati. He did his PhD from Indian Institute of Technology Kanpur and he was involved with an international project (INDO-ITALIAN, N.ER/6), where research on Development of Force Reflecting Surgical Robot Using Micro Fingertip Sensors was undertaken. Further, he was previously engaged in New Delhi Gas Turbine Power Plant as site Engineer recruited by Swamina International Private Limited. Currently he is pursuing collaborative research with Cranfield University on Ionic Polymer Metal Composite applications.

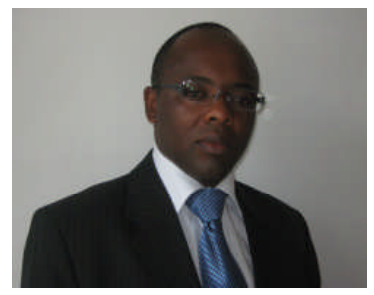

Dr. James Njuguna is an expert in lightweight structures and nanocomposites in the Department of Sustainable Systems at Cranfield University. He is also the Course Director for MSc in Motorsport Engineering and Management Course run at Cranfield University. He holds a PhD in Aeronautical Engineering from City University, London. Dr. Njuguna previously worked as an aircraft engineer before embarking on an academic career. He is a recipient of internationally and nationally recognized awards including a prestigious Research Councils UK Academic Fellowship (2005), Rector of Cracow University of Technology Award (2006) and a Marie Curie Fellowship (2003/04). In addition to significant number of quality scientific journal and conference papers, he is a co-author of a textbook on 'Thermal Degradation of Polymeric Materials' and two book Chapter in nanocomposites. He is a professional member of the Royal Aeronautical Society, Institute of Nanotechnology, American Institute of Aeronautics and Astronautics, and Low Carbon Vehicle Partnership (LowCVP) participating in the Innovations Working Group committee in the latter. 